\title{
Posterior Sub-Tenon Injection of Triamcinolone Acetonide as Pretreatment for Focal Laser Photocoagulation in Diabetic Macular Edema Patients
}

\author{
Chieko Shima $^{1}$, Nahoko Ogata ${ }^{1}$, Keizo Minamino ${ }^{1}$, Tadanobu Yoshikawa ${ }^{1}$, \\ Kayako Matsuyama ${ }^{1}$, and Miyo Matsumura ${ }^{2}$ \\ ${ }^{1}$ Department of Ophthalmology, Kansai Medical University, Moriguchi, Osaka, Japan; \\ ${ }^{2}$ Department of Ophthalmology, Kansai Medical University, Hirakata, Osaka, Japan
}

Japanese Journal of Ophthalmology 2008;52:265-268

The correct name of the fourth author should be given as

Tadanobu Yoshikawa, not Tadanori Yoshikawa. 